\title{
Parkinson's disease: the spectrum of disabilities
}

\begin{abstract}
The needs of people with Parkinson's disease (PD) go beyond the purely medical domain and often require collaborative management. A Panel Discussion at the "Hither neurology" Symposium included neurologists, a speech therapist, a geriatrician and a sociologist. Their discussion highlighted certain aspects of the disability and disadvantage associated with PD. The starting point was a video recording, "Parkinson's Disease: the personal view", in which the contributors were patients and carers. Topics covered included counselling at the time of diagnosis; subsequent access to clinics and to neurological advice; access to therapy; support in the community; fluctuating disability associated with "on-off" phenomena; driving; and sexual problems.
\end{abstract}

Dr Pentland: The most obvious consequences of Parkinson's disease are the impairments: impairments of motor function but also of cognition, emotional state, communication, autonomic function and so on. While neurologists, as a rule, are pretty slick at picking up impairments perhaps they are not quite so adept at detecting and appreciating the extent of the disabilities and handicaps consequent upon these impairments. Do we always realise the full impact of the disorder on the everyday life of the patient and his family?

The Parkinson's Disease Society (PDS) has commissioned an excellent film entitled "Parkinson's disease: the personal view" produced by YEY Television. It was devised by Pauline Smith, Education and Training Officer of the PDS, but is an unscripted and unprompted account by patients and carers of what they feel are matters of concern for them. The perceived needs of patients and carers respectively are summarised in tables 1 and 2 which are taken from the film. This videorecording will act as the basis for the panel discussion.

\section{SEGMENT 1}

$A$ patient, Richard, describes the onset of his symptoms, his initial contacts with his general practitioner and neurologists, the discovery of and his reaction to the diagnosis, his understanding of the likely prognosis and some of the psychological and social consequences of the disease.

Dr Findley: To inform patients of the diagnosis in optimal circumstances you need the right place, person and time. To consider "place" first, the majority of people are told in the outpatient clinic. A few are inpatients and in our experience telling people in the ward or during a ward round is not appropriate. We like to have a relative or carer there with the patient, and we always have a trained counsellor present as well. Adequate time is crucial, avoiding any sense of pressure, so we try to organise our new patient clinic in an area separated from the general hubbub of the rest of outpatient activities. Precisely what information is imparted will depend on the patient's overall abilities, and the approach is essentially an exploration of the individual's knowledge and expectations about the disease. It is usual for us to review the patient four weeks later, so we ask them to think about the diagnosis and write down any questions before this visit. In addition to these consultations, however, the counsellor will see the patient immediately after diagnosis and give him the chance to unload his thoughts and ask questions which he might have felt inhibited about asking the doctor. ${ }^{1}$

Dr Oxtoby: Everything we try to do at Romford is the result of the messages we got from patients about what they wanted and needed. One of the messages from Richard in the video was that he did not feel in control of his life. Our strategy aims to assure people that they have a say in what happens to them right from the beginning. The counsellor is in the room when the doctor explains the diagnosis, hears what is said, sees the patient's reactions, and is able to follow up immediately afterwards. Neurologists tend to feel optimistic about Parkinson's disease and transmit a rather

Table 1 An informal list of patients' needs, from the PDS video "Parkinson's Disease: the personal view"

Needs of Patients

To be treated as individuals

Dignity, respect and independence

Access and early referral to all

appropriate treatment and care

Compassion, understanding, time and patience

Quality of life

Quality of life

Information

To be involved and listened to

A cure

Table 2 An informal list of carers' needs, from the PDS video "Parkinson's Disease: the personal view"

\section{Needs of Carers}

Recognition and acknowledgemen

Information

Honesty, reassurance and encouragement

To be valued and listened to

Practical and emotional help

Access and early referral to all

appropriate treatment and care

A good night's sleep and time for themselve

Understanding and loving respite care

Good health and financial help 
favourable prognosis to the patient. The patient, however, has a different frame of reference and starting point, and needs repeated opportunities to question and seek further information. Discussions should foster a realistic outlook which is a balance of honesty and hopefulness.

Professor Warlow: Has the use of a counsellor been shown to be efficacious, and is it cost effective?

Dr Findley: We have three sources of information on the efficacy of the approach. Firstly, there is what the patients tell us, and they are satisfied. This may not be measurable in objective terms, but is important. Secondly, there are the favourable reports we get back from the local PD support group. Finally, we have looked at a control group of patients in another district where the diagnosis is given in the standard way, whatever that is, and where there is no counsellor service. There are clear differences from our group demonstrating that our approach is better.

We (neurologists) are not trained as counsellors and I think the benefits of a skilled person when imparting serious life-altering diagnoses is enormous. The expenses involved are minuscule. Consider the patient we have just seen on the video; perhaps a whole series of consultations might have been avoided if the diagnosis had been given properly from the start. Thus money is saved in the long term.

Dr Oxtoby: We are not at the stage of costing the service yet. It is relatively easy to measure the cost-benefit of life-saving procedures, but we have a group of patients who are likely to live for a long time, and the important challenge is to improve their quality of life which is not so easily measured. We must also remember that often these patients are unable to express their needs for themselves, and are not demanding. It is our duty as professionals to speak up for them.

Dr Pentland: Loss of the ability to drive can be a devastating handicap. In the film Richard describes losing his licence as though he had lost a relative.

Dr Barnes: One can hardly overestimate the importance of continued driving in the maintenance of independence and even for those who cannot drive it is essential to preserve accessibility to vehicles to ensure mobility outside the home. In Newcastle we have established a Driving Assessment Centre, supported by the PDS. The centre provides advice and information about driving as well as guidance about practical adaptations to vehicles. We are finding that patients receive a great deal of inappropriate advice from neurologists and other health professionals. Cognitive and behavioural difficulties pose more of a problem than physical aspects of fitness to drive. It is very clear that decisions cannot be reliably made on the basis of pencil and paper tests done in the doctor's office. It is also never possible to give advice purely on the results of test rig assessments. There is no substitute for putting the patient behind the wheel of a car with an experienced driving instructor, and trying things out on the road. For those concerned about costs they can be reassured as this service runs at a cost of $£ 25000$ per annum. We have one therapist and a part time secretary, the driving instructors are self-financing as the customer is charged for their services. In fact we hope that by saving on ambulance transport costs the service will become self-financing and cost nothing.

The Royal College of Physicians' report "Physical Disability in 1986 and Beyond"2 recommended driving assessment units as part of each regional rehabilitation centre. Driving assessment is but one example of services which should be concentrated in regional rehabilitation centres. Others might be communication aids centres and advisory services for dysphagia. If we do have such regional centres who should be responsible for running them: neurologists or rehabilitation physicians? There are in fact good arguments for "demedicalisation" of such facilities, that is, for other professionals to manage them, and while in practice it usually depends on the individual personalities involved rather than their specialty. I shall not sit on the fence, but come down on the side of rehabilitation physicians. I would like to see the growth of this separate specialty with its much broader based training programme than that of neurologists. Dr Quinn: If disabled people are to be encouraged to drive there is a need for more adequate hospital parking for disabled people.

\section{SEGMENT 2}

The husband of a young woman with $P D$ describes how different she is when seen in the consultant's clinic, often looking at her best, in contrast to how she may be later that day or the next. He describes how he often thinks "This is when we need the neurologist-to see Valerie now!"

Dr Quinn: The question is again one of time: how much is available and whose time it should be? Parkinson's disease patients, as we all know, take much more time to see in consultation that almost any other diagnostic group. The fact that an effective treatment is available to us compounds the problem as we must discuss manipulating their drugs as well as enquiring how they are. I confess that I often hope the patient will simply say everything is OK, rather than start describing a list of other problems as one is aware of all the other patients queuing up outside.

The other great problem is the fluctuating patient. Personality and mood, as well as motor state, can change dramatically between "on" and "off" states. In these cases one is in effect dealing with two different people in the one individual, and we should meet them both.

How can we improve the management of these patients? An overnight drug withdrawal followed by careful observation of the effects of a single dose of levodopa or apomorphine the following morning can be very valuable. Let me emphasise that I am not promoting hospital admission for drug manipulation. Aside from the enormous cost of hospitalisation already referred to, it is impractical as it takes two weeks for the body to equilibrate after a single 
change in the drug regime of these patients. A brief admission can, however, be very useful in allowing observation of the patient so that we can check that what he describes as "on" and "off" agrees with our concepts of these phenomena and we can determine whether his "shakes" are tremors or dyskinesias. It also provides the opportunity to instruct the patient in keeping good on/off charts.

I do not think neurologists generally have the time to make these observations, and give these instructions but suggest that special nurse practitioners be trained to do so. Such nurse practitioners have been used effectively for several years in the United States for a variety of diseases including Parkinson's disease. They are skilled in the management of specific conditions including prescribing drugs. Indeed, such individuals could go to the patient's home and obviate the need for hospital attendance for observation and treatment.

Dr Findley: I agree with what has been said about the great difficulty resulting from the time constraint in the clinic. The team approach does help. A member of our professional team does a home visit and provides me with a written report before the patient is seen for an annual clinic review.

Dr Oxtoby: It is almost accidental that our "neurocare" team is hospital based and we try to build bridges into the community. ${ }^{3}$ Various people could provide the link: a nurse practitioner, one of the therapists who make up the team or, in certain circumstances, the general practitioner. It is important that someone is given the responsibility or the patient falls between stools.

\section{SEGMENT 3}

Two women who are carers describe the lack of support services to relieve the burden of caring, and explain the need for "emotional and financial back-up".

Dr Pearce: Parkinson's disease is a progressive disorder attended by substantial disabilities requiring support services. Respite and relief for carers is required but in view of the communication problems one must conscientiously pay attention to what the sufferers themselves need too. ${ }^{4}$ It is a family disorder and the aim is to maintain the family unit functioning. The video emphasised the need for emotional support for carers, and I work closely with the clinical psychologist in our clinic to address this aspect. The financial situation of families is another great worry and it is easy for people to slip through the net and be unaware of their entitlements to attendance allowance, mobility allowance and so on. It is worth noting that with the fluctuations in the condition patients may have difficulty getting mobility allowance unless they happen to be immobile on the day the assessor calls.

The nature of the community support system available will differ between rural and urban areas. I am fortunate in practising in a locality with 14 community hospitals, which provide a focus for support which is not identifiably geriatric. Hospital wards are hostile environments for Parkinsonian patients, and I try to restrict respite admissions to two wards rather than scatter them everywhere. These patients need a lot of understanding and tolerance, are at risk of falling, and need a safe environment, have communication problems, and the nursing staff do not get the feedback of gratitude that makes for job satisfaction. Some people reject entirely this form of support, refusing to have their relative admitted to a ward for the elderly. I would like to see people themselves becoming purchasers of services, rather than the authorities determining what form of care they will have.

There is insufficient time to address patients' needs effectively in the clinic, and I believe key workers are necessary. I work with a geriatric liaison nurse who is not fussy about the age spectrum. She has time, and commitment, and has knowledge of all the support services which can be called upon to provide help for these patients. We are also evaluating a key worker system funded by a grant from the Nuffield Provincial Hospital Trust for Young Disabled People.

Finally, although most of Parkinson's disease is dealt with in the community in our district I estimate that patients with the disease continuously occupy eight acute beds which is a considerable consumption of inpatient resources.

\section{SEGMENT 4}

$A$ carer describes the difficulties encountered getting access to appropriate remedial therapy advice stating that, while his general practitioner was generally supportive, he had to make the initiative to have his wife referred to speech and other therapies. His wife makes the plea for doctors to ask and listen to the patient. Another husband relates how people will come up to his wife and himself, and ask him how his wife is, prompting him to tell them "She is here! Ask her!"”

Mrs Scott: It angered but did not surprise me that this man had to go out and find therapy for his wife. It often seems that patients enter a lottery to get speech therapy. Because the patient cannot communicate very well, all too often the neurologist talks to patients rather than discusses things with them and referral to speech therapy does not occur. We might pick them up via the general practitioner but that is very unlikely. ${ }^{56} \mathrm{~A}$ small number come from the local PDS but again that is a case of chance. Often you doctors are not entirely sure what speech therapy can do, but think that on the whole we are nice girls who will at least give the patient a pleasant chat.

Over the last 10 years we have tried very hard to prove what we can do. ${ }^{7-9} \mathrm{We}$ have active roles to play in assessment, management, effective treatment and counselling for the disease; not just the speech problem but the total communication problem that occurs. Some patients are not referred because they are at an early stage and communication difficulties are minimal while at the other extreme some are felt to be beyond our help. In fact we have management 
models tailored to varying degrees of severity of disturbance and by giving short intensive blasts of therapy can help many cases. The situation is also unsatisfactory for occupational therapy although I think it may be a little better in relation to access to physiotherapy.

Question: How does one get the patient, as opposed to the carer, to speak in the clinic?

Mrs Scott: While the carer's account is valuable, sometimes one has to simply say "No, I asked X and want him to answer". If one allows the patient time to answer, he will often be so taken aback that someone wants to listen to him that he will grasp the opportunity and surprise the carer with just how much he does say.

Dr Quinn: As well as there being insufficient neurologists to give enough time to patients with $\mathrm{PD}$, speech therapists also say there are not enough of them for all cases to be referred. Mrs Scott: I agree there are not enough speech therapists, but you must refer the patients and make your therapists cope. We must do this if the need is to be recognised so we can fight with you to improve the service to these patients.

Audience comments: Mr M Hollingworth (YEY Television): As a broadcaster I have found that carers often have an understanding with the patient, and what they say is based on many rehearsals, long before you see them.

Dr D Wilkinson: We constantly hear that we have not got enough time. That is the doctor's problem, not the patient's. If you cannot spend a useful amount of time with a patient, do not see him. As a psychiatrist I like to see both patient and carer separately and together. It is also more effective to see them in their own home.

Dr Pearce: I have a volunteer, a retired psychiatrist, who visits many people before they come to the clinic on the first occasion, and this provides valuable information. The need for home visiting depends partly on the degree of communication there is with the referring doctor.

Professor Marsden: It is important to stress the need to maintain the autonomy of the patient or of the patient and his carer. Someone hinted that the patient and carer had worked out their own way of dealing with the problem in many cases, and we should not interfere with this. In a study of spasmodic torticollis we have recently found that reliance on a "powerful other", by which was meant the doctor, could have a destructive, negative influence on patients coming to terms with their illness and avoiding secondary depression. The major constructive force promoting autonomy was the availability of the patient's own social network in the form of two or three really deep friendships. ${ }^{10}$

Another concern is the manner in which patients, once they reach a certain age, or become incontinent or confused, are shifted from a neurology or medical clinic to the geriatric or psychogeriatric department. What we need are joint clinics by a group of dedicated people to ensure some continuity of care for these families.

Professor Thomas: I am sorry that the discussion has not touched on the role of occupational therapists who, in my experience, often make the most useful contribution.

Dr Ward: Could you comment on sexual problems, which two carers discussed in another segment of the video?

Dr Quinn: We carried out a questionnaire survey and found that about two thirds of male patients and half of their spouses saw themselves as having a sexual problem compared to about one third of female patients and very few of their partners. ${ }^{11}$ The spouse may no longer find their Parkinsonian partner attractive in a sexual sense. Patients can be asked about sexual problems as part of an enquiry about autonomic function. I have never come across anybody who has been offended by my asking. Patients feel it is a very personal aspect of their lives, and the fact that the doctor takes the trouble to ask may actually enhance the doctorpatient relationship. Often one is limited in the advice one can offer, but hopefully the patient can be put in touch with specialist counselling. Even if nothing is available, the fact that the problem is recognised is usually appreciated by the patient.

Dr Pentland: We have seen only a fraction of this excellent film and discussed partially the segments we have seen. We hope the discussions generated, however, have been useful, and will leave you pondering the need to address the wider needs of patients and their families, and consider alternative ways of delivering such a service. Neurologists cannot do it all themselves, but we must assume responsibility for promoting the development of such facilities.

1 Oxtoby M. Telling a patient he has Parkinson's disease. Therapy Weekly 1988;15:4

Royal College of Physicians. Physical Disability in 1986 and Beyond. J Roy Coll Phys Lond 1986;20:319-22.

3 Oxtoby M. Neuro-care needs more therapists. Therapy Weekly 1990;17:11.

4 Gibberd FB, Oxtoby M, Jewell PF. The treatment of Parkinson's disease: a consumer view. Health Trends 1985;17:19-21.

5 Oxtoby M. Parkinson's disease patients and their social needs. Parkinson's Disease Society, 1982.

6 Mutch WJ, Strudwick A, Roy SK, Downie AW. Parkinson's disease: disability, review and therapy. BMJ 1986;293: 675-7.

7 Robertson S, Thompson F. Speech therapy for patients with Parkinson's disease. Br J Dis Communication 1984;19: 213-24.

8 Scott S, Caird FI. Speech therapy for patients with Parkinson's disease. BMJ 1981;283:1088.

9 Caird FI. Rehabilitation in Parkinson's disease. London Chapman and Hall, 1991 .

10 Jahanshahi $M$. Psychosocial factors and depression in torticollis. J Psychosom Res 1991;35:493-507.

11 Brown RG, Jahanshahi M, Quinn N, Marsden CD. Sexual function in patients with Parkinson's disease and their partners. J Neurol Neurosurg Psychiatry 1990;53:480-6. 